\title{
脑胶质瘤动物模型的研究及应用进展
}

\author{
朱惠芳，张远旭，赵旭东* \\ (中国科学院昆明动物研究所, 云南 昆明 650223)
}

\begin{abstract}
摘要: 脑胶质瘤约占中枢神经肿瘤的一半, 临床治疗效果差。尤其是胶质母细胞瘤, 其恶性程度极高, 预后性 差, 是威胁人类健康的主要恶性肿瘤之一, 因此, 选择一种有效的动物模型是研究脑胶质瘤发病机制及其治疗方 法的关键。随着分子生物学、遗传学的发展, 尤其是转基因小鼠, 以及其他越来越多模式生物的出现, 目前已建立 了多种脑胶质瘤动物模型。该文将对目前所建立的各种脑胶质瘤动物模型予以综述。
\end{abstract}

关键词: 脑胶质瘤; 动物模型; 转基因小鼠; 肿瘤干细胞

中图分类号: R739.41；R-332 文献标志码: A 文章编号: 0254-5853-(2012)03-0337-06

\section{Animal models of human glioma: the progress of application and investigation}

\author{
ZHU Hui-Fang, ZHANG Yuan-Xu, ZHAO Xu-Dong* \\ (Kunming Institute of Zoology, Chinese Academy of Science, Kunming 650223, China)
}

\begin{abstract}
The glioma accounts for half of the central nervous tumors, among which the glioblastoma multiforme (GBM) is one of the most aggressive and lethal brain tumors. The difficulties in glioma therapy indicate the need of appropriate animal models for preclinical studies. Benefiting from the development of molecular biology, genetics, and transgenic technology, variable animal models of glioma have been established. These animal models of human glioma are reviewed in this paper.
\end{abstract}

Key words: Glioma; Animal model; Transgenic mice; Tumor stem cell

脑胶质瘤是一类常见的颖内脑肿瘤, 占颖内原 发肿瘤的 35\% 60\%，其中 $50 \%$ 为胶质母细胞瘤。 对脑胶质瘤的临床治疗, 其效果不佳, 预后性差, 是中枢神经脑肿瘤中发病率最高的原发性肿瘤。根 据组织学特性, 脑胶质瘤主要分为星形胶质瘤、少 突神经胶质瘤、室管膜瘤以及混合性胶质瘤等。根 据世界卫生组织的分类系统,根据恶性程度, 脑胶质 瘤可分为 I IV 四个等级。I 和 II 级胶质瘤恶性程 度较低, 如星形胶质瘤; III 和 IV 级胶质瘤恶性程 度很高, 如多型性神经胶母细胞瘤 (glioblastoma multiform, GBM), 平均生存期仅一年左右, 是威胁 人类生命健康最严重的恶性肿瘤之一。目前, 国内 尚缺乏统一的脑肿瘤治疗规范, 当前的首选方案是 手术摘除后辅以放射治疗, 联合使用替莫唑胺/卡
氮芥、替尼泊苷化疗; 有条件的单位正在尝试间质 內放化疗、光动力学治疗、免疫治疗、基因治疗、 神经干细胞治疗等新兴治疗方式; 但大多数治疗方 法都有一定的副作用，明显影响患者的生存质量与 生存期，因此，非常有必要建立合适的胶质瘤动物 模型以研究胶质瘤的发病机制及治疗方案。根据获 得肿瘤的来源, 胶质瘤动物模型可分为诱发性脑胶 质瘤模型、移植脑胶质瘤模型和转基因型脑胶质瘤 模型( Du et al, 2005), 其各自用途不一, 标准各异, 现分别作如下介绍。

\section{1 诱发肿瘤}

人类所患肿瘤的 $80 \%$ 是由环境因素引起的, 动 物的诱发肿瘤与人类肿瘤的发生情况近似，且其成

收稿日期: 2012-01-09; 接受日期: 2012-02-07

基金项目: 自然科学基金面上项目（HUWE1 在卵巢癌发展中的作用）(81171960）

*通信作者(Corresponding author), E-mail: zhaoxudong@mail.kiz.ac.cn 
瘤经过较长的演变过程, 是比较类似于人类肿瘤的 动物模型。根据诱导剂的不同, 脑胶质瘤动物模型 又主要分为化学物质诱发和病毒诱发型。化学物质 诱发的脑胶质瘤动物模型首见于利用甲基胆想制 成丸剂植入小鼠脑内而成功诱发的脑胶质瘤。随后, 又出现了新的致瘤物质, 如烷化剂, 尤其是甲基亚 硝基䐂和乙基亚硝基䐂在中枢神经系统中具有很 高的肿瘤诱发率(Koestner, 1990)。由乙基亚硝基腿 诱发的 Fisher 大鼠脑胶质瘤模型, 联合影像学的应 用，是实验室模拟治疗低级胶质瘤的有效方法 (Kish et al, 2001)。化学物质诱发的胶质瘤模型病程 与人类胶质瘤较为相似，可被用于胶质瘤的移植试 验等, 但由于诱发肿瘤的遗传背景不清楚、种类不 一、性质不稳定等因素, 一定程度上限制了其应用。

通过对人类肿瘤的研究发现, 很多肿瘤的发生 都与病毒相关。病毒诱导肿瘤发生的可能机制有两 个: 其一是病毒通过将其基因组整合至宿主细胞基 因组，导致宿主肿瘤相关基因的激活或抑癌基因的 失活; 其二是有些病毒基因内含有致癌基因, 其编 码的蛋白可促使宿主细胞发生癌变。目前应用于诱 发脑胶质瘤动物模型的病毒有 BKV、JCV 和 RSV 等(Copeland et al, 1976; Krynska et al, 1999; London et al, 1978; Tabuchi et al, 1985)。London et al (1978) 将 JCV 接种至 4 只成年猴子脑内, 结果有 2 只生成 胶质瘤; Tabuchi et al(1985)将 RSV 感染的鸡胚胎成 纤维细胞注射至 15 只猴子脑内, 结果有 11 只生成 脑肿瘤，诱发效率达 $73 \%$ 。病毒诱发模型是用于研 究胶质瘤放/化疗效果的较好模型; 但由于该胶质瘤 模型动物表达某些病毒性肿瘤特异性抗原, 在研究 胶质瘤免疫疗法时, 表现出很强的免疫原性, 因 此, 在实际应用中并不 常用。

总之, 应用化学物质和病毒在动物体内诱发胶 质瘤可在很大程度上模拟自然状态下人类脑胶质 瘤的发生, 但诱发型模型遗传机制不清楚, 生物学 特征不稳定，导致该模型的应用也存在一定的局 限性。

\section{2 移植模型}

移植性动物模型是目前应用最多的肿瘤模型, 也是胶质瘤动物模型中使用最多的方法。随着免疫 缺陷动物, 如裸鼠和 SCID 鼠的出现, 以及准确的 立体定向、严格的无菌操作和浓缩细胞混悬液等技 术的不断完善, 大大提高了移植性动物模型建立的
成功率。

根据肿瘤供体动物与受体动物之间种属的差 异性，移植性肿瘤动物模型可分为异种移植和同种 移植模型：（1）异种移植模型：免疫缺陷小鼠可大 大降低移植过程中动物的免疫排斥反应, 如人类胶 质瘤细胞系 U251 皮下或乑内注射的裸鼠模型，可 模拟人类胶质瘤的典型性特征, 在组织病理学和免 疫组织化学方面与人类胶质母细胞瘤极为相似 (Furnari et al, 2007; Radaelli et al, 2009); 人类胶质 瘤细胞系 U87 颖内注射的裸鼠模型虽然与人类胶 质瘤不太相似，但却是用于研究抗肿瘤血管生成及 胶质瘤高复发率的良好模型(Candolfi et al, 2007; de Vries et al, 2009; Radaelli et al, 2009)。（2）同种移植 模型: 同种移植动物模型的特点是肿瘤组织或细胞 移植发生在同种或同系动物之间。常用的同种移植 性胶质瘤动物模型有: 小鼠胶质瘤细胞系 GL261 颅内注射正常小鼠模型; 大鼠胶质瘤细胞系 C6 接 种于 Long-Evans 大鼠模型 (Branle et al, 2002; Parsa et al, 2000); 大鼠胶质瘤细胞系 9L 脑纹状体 注射Wistar 大鼠模型(Stojiljkovic et al, 2003)。由于 是在正常动物体内建立的肿瘤模型，其肿瘤生长特 性和免疫应答等与人类胶质瘤较为接近, 因此, 应 用该模型获得了很多基于免疫反应的治疗性实验 数据(Maes \& Van Gool, 2011)。

肿瘤干细胞假说的确立以及各种肿瘤干细胞 株的成功分离为肿瘤移植动物模型的建立提供了 新思路。在恶性程度较高的, 如 IV 级胶质瘤中已 经证实了胶质母细胞瘤干细胞存在(Ghods et al, 2007; Kondo et al, 2004; Pellegatta et al, 2006)。根据 肿瘤干细胞的培养特性, 从临床胶质瘤患者所获样 本中已成功分离出胶质母细胞瘤干细胞(Bleau et al, 2008; Galli et al, 2004; Lee et al, 2006; Yuan et al, 2004)。Yuan et al（2004）将从胶质瘤患者中分离得 到的胶质母细胞瘤干细胞接种至裸鼠脑内, 直接导 致了脑肿瘤的发生。此外, 在耐常规放疗和化疗方 面, 胶质母细胞瘤干细胞移植模型也是很好的研究 材料 (Bao et al, 2006; Galli et al, 2004)。胶质母细胞 瘤移植虽然也能够在受体动物中导致胶质瘤的发 生，但该模型不能用于靶向性治疗胶质母细胞瘤。 相比较而言, 胶质母细胞瘤干细胞移植模型能够重 现原癌症的组织学、分子生物学等特征, 能更真实 地还原并反映人类胶质瘤的病理特征与分子生物 学特性。该模型用于研究靶向性杀死胶质母细胞瘤 
干细胞的方法将为治愈胶质母细胞瘤提供了可能 性, 是近年来兴起的一类新型肿瘤动物移植模型。

动物脑肿瘤的移植模型的主要优点是移植瘤 株易存活, 易建立及保存, 且保留了较好的人脑胶 质瘤的组织形态学、分子生物学及细胞生物学特性, 是研究脑肿瘤放/化疗等的较好模型, 但此模型对 研究人脑胶质瘤的遗传机制帮助不大。

\section{3 转基因型}

肿瘤动物模型最早源于小鼠自发突变系或经 致癌物诱导得到, 但由于自发频率在自然状态下 通常很低，且不可精确控制而限制了其应用，而 传统的肿瘤移植模型也并不能充分地模拟人类肿 瘤的发生。随着人们对癌基因激活和抑癌基因失活 在肿瘤发生发展中作用认识的逐步加深, 以及近 年来转基因和基因剔除技术的发展, 转基因肿瘤 动物模型应运而生。

\section{1 转基因胶质瘤动物模型的分子基础}

随着各种癌症相关基因被发现, 人们在基因水 平对肿瘤发生机制的认识也逐步加深, 很多人类肿 瘤的发生通常伴随着癌基因的激活和抑癌基因的 突变或缺失, 胶质瘤的发生也不例外。 $R b$ 和 $p 53$ 是 调控细胞周期和细胞程序性死亡的关键性肿瘤抑 制基因, 在恶性胶质瘤患者中通常可检测到 $R b$ 基 因和 $p 53$ 基因的突变(Watanabe et al, 2001)。这些基 因的失活可表现为 $C d k 4$ 和 $C d k 6$ 基因的扩增和抑癌 基因 $p 16^{I n k 4 a}$ 的失活(Furnari et al, 2007); 而 p53 功能 的缺失还可表现为 $M d m 2$ 或 $M d m 4$ 的扩增, 或抑癌 基因 $p 14^{a r f}$ 的失活(Zhu \& Parada, 2002)。表皮生长 因子受体 EGFR 的持续性激活是恶性胶质瘤发生的 另一特征, EGFR 功能的变化通常表现为该基因的 扩增、点突变和缺失, 其中 EGFRvIII 突变体在恶性 胶质瘤中出现地非常频繁, 激活的 EGFR 通过抑制 $\mathrm{GBM}$ 细胞凋亡并加快其增殖(Aldape et al, 2004)。 血小板来源的生长因子受体 PDGFR 和其配体 PDGF-A 及 PDGF-B 在很多胶质瘤细胞中通常也可 检测到过表达( Zhu \& Parada, 2002)。络氨酸蛋白激 酶(tyrosine protein kinase, PTK)通过激活下游效应 分子 Ras 和 PI $3 \mathrm{~K}$ 从而介导细胞生长和增殖, 而这些 蛋白的活性严格受到肿瘤抑制因子 pTen 和 NF-1 的 调控, 一旦后两者的缺失或突变发生, 将导致胶质 瘤的发生。

近年来, 测序技术在基因组测序中的应用为分
析胶质瘤细胞正常组织细胞中基因的差异提供了 可能。2009 年, TCGA(The Cancer Genome Atlas Network)通过测序分析 206 例人 GBM 病例, 比较样 本中 DNA 拷贝数、基因表达量、DNA 甲基化修饰、 以及基因突变或缺失等, 最终在大多数 $\mathrm{GBM}$ 患者 中发现 $P T K / R a s / P I 3 K, p 53$ 和 $R b$ 的突变, 而 $p 53$ 、 $p T e n 、 E G F R 、 C D K N 2 A / 2 B$ 和 $N F-1$ 是突变最频繁 的基因 ( Cancer Genome Atlas Research Network, 2008)。TCGA 公布的这些数据将为建立转基因胶质 瘤动物模型提供更多的选择和可能性, 而这些动物 模型系统的建立又将更好地为研究胶质瘤的发生 发展机制以及临床前期的试验服务。

\section{2 转基因胶质瘤动物模型的建立方法}

小鼠是最主要的转基因模式动物, 被广泛的 应用于生物科学和医药研究中。利用转基因技术已 经建立了成熟的小鼠胶质瘤模型。这些转基因小鼠 通过不同癌基因和抑癌基因的组合，实现肿瘤基因 在小鼠中的过表达和抑癌基因的灭活，从而导致胶 质瘤的发生。目前采用的方法主要有两大类：（1） 传统的转基因小鼠类型; (2) 病毒载体介导类型。

建立在受精卵和胚胎干细胞基础上的基因导 入或敲除技术建立了很多动物肿瘤模型。然而, 这 些肿瘤模型与人类肿瘤仍然存在很大差异, 如人类 肿瘤是在出生后, 随着环境因素的影响而发病的, 而传统的转基因动物肿瘤模型是在胚胎发育期间 人为改变癌基因或抑癌基因的表达水平, 其肿瘤的 发生比人类肿瘤实际发生的时间要早, 并且肿瘤发 生的动态学变化与人类肿瘤也不尽相同, 因此, 需 要引进新的技术以建立与人类肿瘤更为接近的动 物肿瘤模型。Cre-loxP 技术在动物肿瘤模型中的运 用正是为解决这些问题而发展起来的。Zhu et al (2005)通过 Cre-loxp 技术实现了条件性基因敲除小 鼠胶质瘤模型, 通过 Cre 重组酶介导抑癌基因 NF-1 和 p53 的缺失, 胶质纤维酸性蛋白 (glial fibrillary acidic protein, GFAP)启动子控制基因只能在特异性 的胶质细胞中表达, 该小鼠 (GFAP-cre/NF$1^{f /+} / p 53^{-/+}$) 模型可条件性控制后天性胶质瘤的发生 (早期诱导 II 级胶质瘤, 随后发展为 III 级胶质瘤, 最后发展为 IV 级)。Kwon et al (2008)发现, 在 $G F A P$-cre $/ N F-1^{f /+} / p 53^{-/+}$小鼠基础上, 条件性敲除 $p T e n\left(G F A P-\operatorname{cre} / N F-1^{f /+} / p 53^{-/+} / p T e n^{f /+}\right)$, 可直接诱导 $\mathrm{IV}$ 级恶性胶质瘤的发生。随着实验技术的不断更新, 之后又出现了 Nestin-CreER介导的特异性靶向神经 
干细胞的胶质瘤小鼠模型 Nestin-creER/ $N F-1^{f /+} / p 53^{-/+} / p T e n^{f /+}$ 。Nestin 蛋白是神经干细胞的 特征性分子标记, 在他莫昔芬(tamoxifen)处理下, 可使 CreER 进入细胞核内, 介导 loxp 位点的重组, 在新生小鼠及成年小鼠的脑室下区(sub-ventricular zone, SVZ)可诱导肿瘤的 100\%发生(分别为 12/12、 12/12)(Chen et al, 2009; Llaguno et al, 2009)。采用转 基因的方法, 通过不同癌基因和抑癌基因的组合, 可建立不同类型的小鼠脑胶质瘤模型(Sughrue et al, 2009), 表 1 总结了目前主要的几种转基因胶质瘤 模型。

表 1 转基因胶质瘤小鼠模型

Tab. 1 The glioma model of transgeneic mice

\begin{tabular}{|c|c|c|}
\hline $\begin{array}{c}\text { 肿瘤类型 } \\
\text { Tumor classification }\end{array}$ & $\begin{array}{l}\text { 基因表型 } \\
\text { Genetics }\end{array}$ & $\begin{array}{l}\text { 参考文献 } \\
\text { Reference }\end{array}$ \\
\hline $\begin{array}{l}\text { High-grade } \\
\text { astrocytoma }\end{array}$ & $\begin{array}{l}\text { RasB8 mice based- } \\
\text { EGRFvIII/pTen }\end{array}$ & Wei et al, 2006 \\
\hline Low-grade astrocytoma & EGRFvIII/pTen ${ }^{-/-}$ & Li et al, 2009 \\
\hline High-grade glioma & EGFRvIII/Ink4a/Arf ${ }^{/-}$ & Bachoo et al, 2002 \\
\hline GBM & EGFRvIII/Ink4a/Arf ${ }^{1-} / \mathrm{pTen}^{-/-}$ & Zhu et al, 2009 \\
\hline Malignant astrocytoma & $\mathrm{NF}-1^{+/-} / \mathrm{p} 53^{+/-}$ & Reilly et al, 2000 \\
\hline $\begin{array}{l}\text { High-grade } \\
\text { astrocytoma }\end{array}$ & $\mathrm{NF}-1^{+/-} / \mathrm{p} 53^{+/-} / \mathrm{pTen}^{-/-}$ & Kwon et al, 2008 \\
\hline Oligodendroglioma & v-erbB/EGFRvIII & Weiss et al, 2003 \\
\hline Oligodendroglioma & v-Ras/EGFRvIII & Ding et al, 2003 \\
\hline
\end{tabular}

病毒介导的胶质瘤模型, 根据使用载体的不同 又可分为腺病毒介导(Ahmed et al, 2011; Llaguno et al, 2009; Zhu et al, 2009)、逆转录病毒介导( Li et al, 2009; Uhrbom \& Holland, 2001)和慢病毒介导(de Vries et al, 2010; Marumoto et al, 2009)胶质瘤模型。 三种载体各有其优缺点: 腺病毒载体具有高效传递 和表达基因的能力, 但腺病毒自身具有的非特异致 瘤性限制了其应用; 逆转录病毒基因组能稳定地整 合至宿主基因组中, 但其宿主范围较窄, 只能感染 分裂细胞; 慢病毒载体是目前应用前景较好的转基 因载体, 该载体不仅能将外源基因有效地整合到靶
细胞基因组内, 而且可以感染各种非分裂细胞。 Holland et al (2000)通过逆转录病毒 RCAS-TVA 系 统激活小鼠大脑中 Ras 和 $A k t$ 的表达, 可实现目标 基因在胶质细胞中的靶向性表达; Marumoto et al (2009)通过慢病毒载体介导 Ras 的表达或 $A k t$ 的激 活，它们的表达受到 Cre 重组酶的控制, 将病毒注 入 GFAP-Cre 基因小鼠海马区或脑室下区 (sub-ventricular zone, SVZ), 可导致 GBM 的发生, 但如果将病毒注射在大脑皮层，则无法产生脑胶质 瘤; Jacques et al(2010)通过腺病毒载体 GFAP-Cre 系 统灭活 $R b 、 p 53$ 和（或） pTen 基因在 GFAP 表达的 胶质细胞中表达，同样也建立了胶质瘤的小鼠模 型。

运用转基因方法建立的胶质瘤小鼠模型，可按 照人们意愿在分子水平和细胞水平对动物的遗传 物质进行改变, 动物遗传背景清楚, 可模拟出临床 上由各类因素引起的基因变异而导致的胶质瘤，在 病因学上更接近于脑胶质瘤的自然发生过程, 为肿 瘤的发生、发展及治疗提供了良好的研究材料。

\section{4 讨论与展望}

肿瘤的发生往往是由于染色体畸变、碱基缺失 或突变, 或者肿瘤相关基因表观修饰改变导致。 TCGA 通过对胶质母细胞瘤患者样本的分析发现, 大多数胶质母细胞瘤患者的肿瘤组织中存在 $P T K / R a s / P I 3 K 、 p 53$ 和 $R b$ 等基因的突变, 而 $p 53$ 、 pTen、EGFR、CDKN $2 A / 2 B$ 和 $N F-1$ 是突变最频繁 的基因。正是这些基因突变的发现, 为研究者建立 转基因胶质瘤动物模型提供了重要的分子基础。

目前建立脑胶质瘤的方法有诱发型、移植型和 转基因型三类，虽然前两种方法的运用时间较长， 获得的动物模型目前使用比较广泛，但却各有其局 限性而限制了它们的应用范围。各种模型的适用范 围及优缺点见表 2 。相对来说, 转基因动物模型具

表 2 三种肿瘤动物模型优缺点比较

Tab. 2 The comparison of three animal tumor models

\begin{tabular}{|c|c|c|c|c|}
\hline $\begin{array}{c}\text { 模型分类 } \\
\text { Classification }\end{array}$ & $\begin{array}{c}\text { 亚类 } \\
\text { Sub-classification } \\
\end{array}$ & $\begin{array}{c}\text { 适用范围 } \\
\text { Application }\end{array}$ & $\begin{array}{c}\text { 优点 } \\
\text { Advantage } \\
\end{array}$ & $\begin{array}{c}\text { 缺点 } \\
\text { Disadvantage }\end{array}$ \\
\hline \multirow{2}{*}{ 诱导模型 } & 化学诱导 & 移植试验 & \multirow{2}{*}{ 病程与人类肿瘤相似 } & \multirow{2}{*}{ 遗传背景不清楚 } \\
\hline & 病毒诱导 & 肿瘤的放疗化疗 & & \\
\hline \multirow{2}{*}{ 移植模型 } & 同种移植 & 抗肿瘤新药篮选 & 瘤株易以保存 & \multirow{2}{*}{ 肿瘤生长不稳定 } \\
\hline & 异种移植 & 肿瘤的放疗化疗 & 一致性较好 & \\
\hline \multirow{2}{*}{ 转基因模型 } & 转基因小鼠 & 临床抗肿瘤药物的药物评估 & 遗传背景清楚, 分子机制明确 & \multirow{2}{*}{$\begin{array}{c}\text { 基因拷贝数不可控 } \\
\text { 组织特异性注射难 } \\
\text { 度教大 }\end{array}$} \\
\hline & 病毒载体介导 & 肿瘤的免疫疗法及疫苗研究 & 表达系统稳定, 免疫原性小 & \\
\hline
\end{tabular}


有分子机制明确、建立系统稳定、重复性好等优点， 随着分子生物学技术的不断完善, 转基因动物胶质 瘤动物模型的优势体现得越来越充分，尤其是慢病 毒介导的胶质瘤动物模型, 其载体构建简单, 表达 系统稳定性强, 免疫原性小, 是一类很有发展潜力 的转基因方法。转基因动物模型缺点是组织特异性 注射难度比较大, 而靶向转基因小鼠因具有其独特 的优势，因此，需结合两种方法使用。

脑胶质瘤动物模型的运用为临床上治疗人类 胶质瘤的前期研究提供了重要信息, 虽然动物模型

\section{参考文献:}

A hmed AU, Thaci B, Alexiades NG, Han Y, Qian S, Liu FF, Balyasnikova IV, Ulasov IY, Aboody KS, Lesniak MS. 2011. Neural stem cell-based cell carriers enhance therapeutic efficacy of an oncolytic adenovirus in an orthotopic mouse model of human glioblastoma[J]. Mol Ther, 19(9): 1714-1726.

Aldape KD, Ballman K, Furth A, Buckner JC, Giannini C, Burger PC, Scheithauer BW, Jenkins RB, James CD. 2004. Immunohistochemical detection of EGFRvIII in high malignancy grade astrocytomas and evaluation of prognostic significance[J]. J Neuropathol Exp Neurol, 63(7): 700-707.

Bachoo RM, Maher EA, Ligon KL, Sharpless NE, Chan SS, You MJ, Tang Y, DeFrances J, Stover E, Weissleder R, Rowitch DH, Louis DN, DePinho RA. 2002. Epidermal growth factor receptor and Ink4a/Arf: convergent mechanisms governing terminal differentiation and transformation along the neural stem cell to astrocyte axis[J]. Cancer Cell, 1(3): 269-277.

Bao SD, Wu QL, McLendon RE, Hao YL, Shi Q, Hjelmeland AB, Dewhirst MW, Bigner DD, Rich JN. 2006. Glioma stem cells promote radioresistance by preferential activation of the DNA damage response[J]. Nature, 444(7120): 756-760.

Bleau AM, Howard BM, Taylor LA, Gursel D, Greenfield JP, Lim Tung HY, Holland EC, Boockvar JA. 2008. New strategy for the analysis of phenotypic marker antigens in brain tumor-derived neurospheres in mice and humans[J]. Neurosurg Focus, 24(3-4): E28.

Branle F, Lefranc F, Camby I, Jeuken J, Geurts-Moespot A, Sprenger S, Sweep F, Kiss R, Salmon I. 2002. Evaluation of the efficiency of chemotherapy in in vivo orthotopic models of human glioma cells with and without 1p19q deletions and in C6 rat orthotopic allografts serving for the evaluation of surgery combined with chemotherapy[J]. Cancer, 95(3): 641-655.

Candolfi M, Curtin JF, Nichols WS, Muhammad AG, King GD, Pluhar GE, McNiel EA, Ohlfest JR, Freese AB, Moore PF, Lerner J, Lowenstein PR, Castro MG. 2007. Intracranial glioblastoma models in preclinical neuro-oncology: neuropathological characterization and tumor progression[J]. J Neurooncol, 85(2): 133-148.

Cancer Genome Atlas Research Network. 2008. Comprehensive genomic characterization defines human glioblastoma genes and core pathways[J]. Nature, 455(7216): 1061-1068.

Chen J, Kwon CH, Lin L, Li YJ, Parada LF. 2009. Inducible site-specific recombination in neural stem/progenitor cells[J]. Genesis, 47(2): 122-131.

Copeland DD, Talley FA, Bigner DD. 1976. The fine structure of intracranial neoplasms induced by the inoculation of avian sarcoma
能在很大程度上模拟人类胶质瘤的发生，但目前的 研究结果表明, 动物肿瘤模型与人类胶质瘤的发病 机理始终有所差异，在抗肿瘤药物篮选上，很多抗 肿瘤药物对模式胶质瘤动物有很好的疗效，但在人 体上却收效甚微。这就提醒着我们还需要不断开发 建立动物脑胶质瘤模型的新技术，研制出更高质量 的动物脑胶质瘤模型，例如胶质瘤猴模型，在建立 动物模型时考虑人胶质瘤的细胞起源和癌症基因 组学进展等, 使动物脑胶质瘤模型尽量接近人类胶 质瘤发生，最终攻克胶质瘤这一顽症。

virus in neonatal and adult rats[J]. Am J Pathol, 83(1): 149-176.

de Vries NA, Beijnen JH, van Tellingen O. 2009. High-grade glioma mouse models and their applicability for preclinical testing $[\mathrm{J}]$. Cancer Treat Rev, 35(8): 714-123.

de Vries NA, Bruggeman SW, Hulsman D, de Vries HI, Zevenhoven J, Buckle T, Hamans BC, Leenders WP, Beijnen JH, van Lohuizen M, Berns AJM, van Tellingen O. 2010. Rapid and robust transgenic high-grade glioma mouse models for therapy intervention studies[J]. Clin Cancer Res, 16(13): 3431-3441.

Ding H, Shannon P, Lau N, Wu XL, Roncari L, Baldwin RL, Takebayashi H, Nagy A, Gutmann DH, Guha A. 2003. Oligodendrogliomas result from the expression of an activated mutant epidermal growth factor receptor in a RAS transgenic mouse astrocytoma model[J]. Cancer Res, 63(5): 1106-1113.

$\mathrm{Du}$ W, Chen MQ, Wu ZP. 2005. The research progress of mice tumor model[J]. Chn J Pract Med, 4(11): 1120-1122. [杜伟，陈明清，吴治平. 2005. 建立小鼠肿瘤模型的研究进展 $[\mathrm{J}]$. 中华医学实践杂志, 4(11): 1120-1122. ]

Furnari FB, Fenton T, Bachoo RM, Mukasa A, Stommel JM, Stegh A, Hahn WC, Ligon KL, Louis DN, Brennan C, Chin L, DePinho RA, Cavenee WK. 2007. Malignant astrocytic glioma: genetics, biology, and paths to treatment[J]. Genes Dev, 21(21): 2683-2710.

Galli R, Binda E, Orfanelli U, Cipelletti B, Gritti A, De Vitis S, Fiocco R, Foroni C, Dimeco F, Vescovi A. 2004. Isolation and characterization of tumorigenic, stem-like neural precursors from human glioblastoma[J]. Cancer Res, 64(19): 7011-7021.

Ghods AJ, Irvin D, Liu GT, Yuan XP, Abdulkadir IR, Tunici P, Konda B, Wachsmann-Hogiu S, Black KL, Yu JS. 2007. Spheres isolated from 9L gliosarcoma rat cell line possess chemoresistant and aggressive cancer stem-like cells[J]. Stem Cells, 25(7): 1645-1653.

Holland EC, Celestino J, Dai CK, Schaefer L, Sawaya RE, Fuller GN. 2000. Combined activation of Ras and Akt in neural progenitors induces glioblastoma formation in mice[J]. Nat Genet, 25(1): 55-57.

Jacques TS, Swales A, Brzozowski MJ, Henriquez NV, Linehan JM, Mirzadeh Z, O'Malley C, Naumann H, Alvarez-Buylla A, Brandner S. 2010. Combinations of genetic mutations in the adult neural stem cell compartment determine brain tumour phenotypes[J]. EMBO J, 29(1): 222-235.

Kish PE, Blaivas M, Strawderman M, Muraszko KM, Ross DA, Ross BD, McMahon G. 2001. Magnetic resonance imaging of ethyl-nitrosourea-induced rat gliomas: a model for experimental therapeutics of low-grade gliomas[J]. J Neurooncol, 53(3): 243-257.

Koestner A. 1990. Characterization of N-nitrosourea-induced tumors of the 
nervous system; their prospective value for studies of neurocarcinogenesis and brain tumor therapy[J]. Toxicol Pathol, 18(2): 186-192.

Kondo T, Setoguchi T, Taga T. 2004. Persistence of a small subpopulation of cancer stem-like cells in the C6 glioma cell line[J]. Proc Natl Acad Sci USA, 101(3): 781-786.

Krynska B, Otte J, Franks R, Khalili K, Croul S. 1999. Human ubiquitous $\mathrm{JCV}(\mathrm{CY}) \mathrm{T}$-antigen gene induces brain tumors in experimental animals[J]. Oncogene, 18(1): 39-46.

Kwon CH, Zhao DW, Chen J, Alcantara S, Li YJ, Burns DK, Mason RP, Lee EYHP, Wu H, Parada LF. 2008. Pten haploinsufficiency accelerates formation of high-grade astrocytomas $[\mathrm{J}]$. Cancer Res, $\mathbf{6 8}(9)$ : 3286-3294.

Lee J, Kotliarova S, Kotliarov Y, Li AG, Su Q, Donin NM, Pastorino S, Purow BW, Christopher N, Zhang W, Park JK, Fine HA. 2006. Tumor stem cells derived from glioblastomas cultured in bFGF and EGF more closely mirror the phenotype and genotype of primary tumors than do serum-cultured cell lines[J]. Cancer Cell, 9(5): 391-403.

Li L, Dutra A, Pak E, Labrie JE III, Gerstein RM, Pandolfi PP, Recht LD, Ross AH. 2009. EGFRvIII expression and PTEN loss synergistically induce chromosomal instability and glial tumors[J]. Neuro Oncol, 11(1): 9-21.

Llaguno SA, Chen J, Kwon CH, Jackson EL, Li YJ, Burns DK, Alvarez-Buylla A, Parada LF. 2009. Malignant astrocytomas originate from neural stem/progenitor cells in a somatic tumor suppressor mouse model[J]. Cancer Cell, 15(1): 45-56.

London WT, Houff SA, Madden DL, Fuccillo DA, Gravell M, Wallen WC, Palmer AE, Sever JL, Padgett BL, Walker DL, ZuRhein GM, Ohashi T. 1978. Brain tumors in owl monkeys inoculated with a human polyomavirus (JC virus)[J]. Science, 201(4362): 1246-1249.

Maes W, Van Gool SW. 2011. Experimental immunotherapy for malignant glioma: lessons from two decades of research in the GL261 model[J]. Cancer Immunol Immunother, 60(2): 153-160.

Marumoto T, Tashiro A, Friedmann-Morvinski D, Scadeng M, Soda Y, Gage FH, Verma IM. 2009. Development of a novel mouse glioma model using lentiviral vectors[J]. Nat Med, 15(1): 110-116.

Parsa AT, Chakrabarti I, Hurley PT, Chi JH, Hall JS, Kaiser MG, Bruce JN. 2000. Limitations of the C6/Wistar rat intracerebral glioma model: implications for evaluating immunotherapy[J]. Neurosurgery, 47(4): 993-1000.

Pellegatta S, Poliani PL, Corno D, Menghi F, Ghielmetti F, Suarez-Merino B, Caldera V, Nava S, Ravanini M, Facchetti F, Bruzzone MG, Finocchiaro G. 2006. Neurospheres enriched in cancer stem-like cells are highly effective in eliciting a dendritic cell-mediated immune response against malignant gliomas[J]. Cancer Res, 66(21): 10247-10252.

Radaelli E, Ceruti R, Patton V, Russo M, Degrassi A, Croci V, Caprera F,
Stortini G, Scanziani E, Pesenti E, Alzani R. 2009. Immunohistopathological and neuroimaging characterization of murine orthotopic xenograft models of glioblastoma multiforme recapitulating the most salient features of human disease[J]. Histol Histopathol, 24(7): 879-891.

Reilly KM, Loisel DA, Bronson RT, McLaughlin ME, Jacks T. 2000. Nf1;Trp53 mutant mice develop glioblastoma with evidence of strain-specific effects[J]. Nat Genet, 26(1): 109-113.

Stojiljkovic M, Piperski V, Dacevic M, Rakic L, Ruzdijic S, Kanazir S. 2003. Characterization of 9L glioma model of the Wistar rat[J]. J Neurooncol, 63(1): 1-7.

Sughrue ME, Yang I, Kane AJ, Rutkowski MJ, Fang SN, James CD, Parsa AT. 2009. Immunological considerations of modern animal models of malignant primary brain tumors[J]. J Transl Med, 7(1): 84.

Tabuchi K, Nishimoto A, Matsumoto K, Satoh T, Nakasone S, Fujiwara T, Ogura H. 1985. Establishment of a brain-tumor model in adult monkeys[J]. J Neurosurg, 63(6): 912-916.

Uhrbom L, Holland EC. 2001. Modeling gliomagenesis with somatic cell gene transfer using retroviral vectors[J]. J Neurooncol, 53(3): 297-305.

Watanabe T, Yokoo H, Yokoo M, Yonekawa Y, Kleihues P, Ohgaki H. 2001. Concurrent inactivation of RB1 and TP53 pathways in anaplastic oligodendrogliomas[J]. J Neuropathol Exp Neurol, 60(12): 1181-1189.

Wei QX, Clarke L, Scheidenhelm DK, Qian BP, Tong A, Sabha N, Karim Z, Bock NA, Reti R, Swoboda R, Purev E, Lavoie JF, Bajenaru ML, Shannon P, Herlyn D, Kaplan D, Henkelman RM, Gutmann DH, Guha A. 2006. High-grade glioma formation results from postnatal pten loss or mutant epidermal growth factor receptor expression in a transgenic mouse glioma model[J]. Cancer Res, 66(15): 7429-7437.

Weiss WA, Burns MJ, Hackett C, Aldape K, Hill JR, Kuriyama H, Kuriyama N, Milshteyn N, Roberts T, Wendland MF, DePinho R, Israel MA. 2003. Genetic determinants of malignancy in a mouse model for oligodendroglioma[J]. Cancer Res, 63(7): 1589-1595.

Yuan XP, Curtin J, Xiong YZ, Liu GT, Waschsmann-Hogiu S, Farkas DL, Black KL, Yu JS. 2004. Isolation of cancer stem cells from adult glioblastoma multiforme[J]. Oncogene, 23(58): 9392-9400.

Zhu HH, Acquaviva J, Ramachandran P, Boskovitz A, Woolfenden S, Pfannl R, Bronson RT, Chen JW, Weissleder R, Housman DE, Charest A. 2009. Oncogenic EGFR signaling cooperates with loss of tumor suppressor gene functions in gliomagenesis[J]. Proc Natl Acad Sci USA, 106(8): 2712-2716.

Zhu Y, Guignard F, Zhao DW, Liu L, Burns DK, Mason RP, Messing A, Parada LF. 2005. Early inactivation of p53 tumor suppressor gene cooperating with NF1 loss induces malignant astrocytoma[J]. Cancer Cell, 8(2): 119-130.

Zhu Y, Parada LF. 2002. The molecular and genetic basis of neurological tumours[J]. Nat Rev Cancer, 2(8): 616-626. 